\title{
Chinese Classical Prescription(Liang-Xue-Di-Huang Decoction)for Hemorrhoidal Disease: study protocol for a randomized controlled trial
}

Qing Zhou (D 13601401869@163.com)

Nanjing university of chinese medicine https://orcid.org/0000-0002-5432-3652

Shuo-yang Shi

Nanjing University of Chinese Medicine

Zong-qi He

Nanjing University of Chinese Medicine

Cheng-biao Xu

Nanjing University of Chinese Medicine

Ji Geng

Nanjing University of Chinese Medicine

Tuo Chen

Nanjing University of Chinese Medicine

Zhao-feng Shen

Nanjing University of Chinese Medicine

Dan Zhang

Nanjing University of Chinese Medicine

Feng Jiang

Nanjing University of Chinese Medicine

Yu-Gen Chen

Nanjing University of Chinese Medicine

Ben-sheng Wu

Nanjing University of Chinese Medicine

\section{Study protocol}

Keywords: Hemorrhoidal disease, Liang-Xue-Di-Huang Decoction, Chinese classical prescription, Randomized controlled trial

Posted Date: August 23rd, 2019

DOI: https://doi.org/10.21203/rs.2.13478/v1 
License: (c) (i) This work is licensed under a Creative Commons Attribution 4.0 International License. Read Full License 


\section{Abstract}

Abstract Background: Hemorrhoidal disease (HD) is one of the commonest proctologic condition in the general population. Medical therapy for HD has not been formally confirmed due to the inconsistent of results. Liang-Xue-Di-Huang Decoction, a kind of ancient Chinese classical prescription, has been used to treat HD from the 19th century in China. However, clinical research of Liang-Xue-Di-Huang Decoction in the treatment of HD is lack. We designed this study to evaluate the efficacy and safety of Liang-Xue-DiHuang Decoction in the treatment of HD. Methods/Design: A randomized, controlled, double blind, double-mimetic agent and multicenter trial to evaluate the efficacy and safety of Liang-Xue-Di-Huang Decoction is proposed. HD patients (stage I, $\triangle, \bigotimes$ ) will be randomly assigned into Liang-Xue-Di-Huang Decoction with the addition of Diosmine mimetic agent group and Diosmine with the addition of LiangXue-Di-Huang Decoction mimetic agent group. Patients will receive a 7-day treatments and a 7-day follow-up. The primary outcome measure is the French Bleeding Score in 7 and 14 days. The Secondary outcome measures are Goligher Prolapse Score and Quality-of-Life Score in 7 and 14 days. Discussion: This study will provide objective evidence to evaluate the efficacy and safety of Liang-Xue-Di-Huang Decoction in treatment of HD. Trial registration: Chinese Clinical Trial Registry. ChiCTR1900022531.Registered 15 Apr 2019, http://www.chictr.org.cn/listbycreater.aspx.

\section{Background}

Hemorrhoidal disease (HD), one of the oldest and commonest proctologic condition in the general population, is more frequent in industrialized countries ${ }^{[1]}$. The true prevalence of $\mathrm{HD}$ in the general population is unknown and probably differents from country to country ${ }^{[2]}$. In the United States, HD is the third most common outpatient gastrointestinal diagnosis, affecting between 20 to $50 \%$ of the population and resulting in 4 million emergency visits annually ${ }^{[3]}$.

HD exists in two forms-an external and an internal disease. External hemorrhoids are laid below the dentate line. Internal hemorrhoids are located above the dentate line, which are characterized by dilatation and engorgement of the hemorrhoidal plexuses and vascular hyperplasia. Chronic bleeding is the main symptom of internal hemorrhoids ${ }^{[4]}$.

Treatments for HD include medical therapies and surgery ${ }^{[5]}$. Medical therapies for HD have not been formally studied where the results have been inconsistent ${ }^{[3]}$. Increased fiber and fluid intake has been shown to improve symptoms of mild-to-moderate bleeding, whereas symptoms such as prolapse and pain show a tendency toward no effect ${ }^{[6]}$. The fiber is not recommended as primary treatment of moderate-to-severe prolaps $\mathrm{e}^{[7]}$. Another common medical prescription in patients with bleeding hemorrhoids is micronized purified flavonoid fraction. These drugs include diosmin, hesperidin, and cumarin. Each of these treatments has advantages and drawbacks, which are mild gastrointestinal disturbances $^{[2]}$. There is still no evidence from well-designed studies to support the use of any of the overthe-counter preparations ${ }^{[8]}$. 
Liang-Xue-Di-Huang Decoction, a kind of Chinese herbal medicine, listed in Table 1, which has been approved by the National Administration of Traditional Chinese Medicine of People's Republic of China in

$2018^{[9]}$, has been used for HD from the 19th century in China with good effects and few adverse events. However, it was still necessary to prove the efficacy and safety of this ancient Chinese classical prescription. The aim of the present study is to evaluate Liang-Xue-Di-Huang Decoction in the treatment of HD using a randomized, controlled, blind and multicenter trial among officially registered Chinese colorectal consultants, fellows and residents in China.

\section{Methods}

\section{Design}

This study is designed as a randomized, controlled, blind and multicenter trial. Trained researchers introduce the trial to patients, give them information sheets and consent forms. All patients have to obtain "Ethics approval and consent to participate" section and give their written informed consents prior to enrolment. The study's flow chart is shown in Figure 1.

\section{Ethics}

The trial protocol is conducted in accordance with the Good Clinical Practice Guidelines and the Declaration of Helsinki (2008) ${ }^{[10]}$. Central ethical approval has been confirmed from the group leader's ethic committee of Affiliated Hospital of Nanjing University of Chinese Medicine (ref approval no. 2019NL-158-02) and four sub-centers ethical will comply with the group leader's ethics approval. Written informed consent will be obtained from each patient.

\section{Recruitment}

A total of 240 Chinese patients who fulfill the screening criteria will be recruited at five hospitals in China:

1) Group leader, Affiliated Hospital of Nanjing University of Chinese Medicine, will recruit 64 patients through posters, 2) Sub-center, Changzhou affiliated Hospital of Nanjing University of Chinese Medicine, will recruit 60 patients through posters, 3) Sub-center, Suzhou affiliated Hospital of Nanjing University of Chinese Medicine, will recruit 60 patients through posters, 4) Sub-center, Xuyi affiliated Hospital of Nanjing University of Chinese Medicine, will recruit 28 patients through posters, 5) Sub-center, Wujin affiliated Hospital of Nanjing University of Chinese Medicine, will recruit 28 patients through posters.

\section{Sample size}

According to the literature search, bleeding hemorrhoids was used as the scoring criteria for the treatment of HD. It is expected that the use of Liang-Xue-Di-Huang Decoction treatment is not inferior to diosmine treatment. For a two-sided significance level of 0.05 and power of $80 \%(a=0.05, \beta=0.2, \delta=0.15)$, the sample size is calculated using the formula: 


$$
\mathrm{n}_{\mathrm{T}}=\frac{2\left(z_{\alpha / 2}+z_{\beta}\right)^{2} \sigma^{2}}{\delta^{2}}
$$

Considering a $10 \%$ loss to follow-up, the sample size is 240 cases ( $n=120$ in each group).

\section{Randomization}

Block randomization was used. Stratification was carried out according to the center. With the help of SAS 9.4 statistical software, the random number table of central coding was generated for a given number of seeds. The subjects were randomly divided into Liang-Xue-Di-Huang Decoction with the addition of Diosmine mimetic agent group, and Diosmine with the addition of Liang-Xue-DiHuang Decoction mimetic agent group. An independent person (Zhao-feng Shen), who is not involved in observation or assessment of the patients possesses the computer-generated randomization sequence.

The randomization procedure will be conducted by research assistants using an online computerized randomization system (https://sci.medroad.cn/).

\section{Blinding}

This trial is a double-blind trial, divided into the Liang-Xue-Di-Huang Decoction with the addition of Diosmine mimetic agent group,and Diosmine with the addition of Liang-Xue-Di-Huang Decoction mimetic agent group. The double levels of blinding are sealed separately, and given to the leader and the sponsor of the clinical research. Each hospital receives an emergency letter, along with these test drugs, properly preserved until the end of the trial. Treatments are blinded to the patients and investigators (including statisticians) until the entire study is completed.

Code-breaking should occur only in the case of serious adverse events happen or further intervention of the patient needs to know the actual medication situation, with the permission of the person in charge of the research center, and a report should be submitted to the leader of the trial within 24 hours.

\section{Eligibility criteria}

\section{Inclusion criteria:}

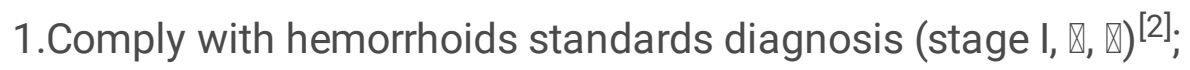

2.Comply with "Traditional Chinese Medicine Disease and Syndrome, Diagnosis and Curative Effect Standard" damp-heat syndrome: bleeding hemorrhoids, bright red blood ${ }^{[11]}$;

3.Patient signed the informed consent form;

4.Patient agreed to avoid taking study agents outside the trial;

\section{Exclusion criteria:}


1.Patient had accompanied by severe liver, kidney, heart, brain, or lung dysfunction;

2.Patient had a history of inflammatory bowel disease, or a history of colorectal cancer, or had a history of any cancer;

4.Patient had a perianal abscess, anal fistula, rectal polyp, intestinal tumor or intestinal infectious disease;

5.Patient will plan pregnancy during this study;

6.Patient is pregnant or lactating women at the time;

7.Patient was allergic to test drugs and their ingredients;

8.Patient had unability to understand the nature of the study and follow the doctor's recommendations.

\section{Test drugs}

Test drugs are Liang-Xue-Di-Huang Decoction, Liang-Xue-Di-Huang Decoction mimetic agent, Diosmine and Diosmine mimetic agent. Liang-Xue-Di-Huang Decoction and Liang-Xue-Di-Huang Decoction mimetic agent are provided by Tianjiang Pharmaceutical Group Co. Ltd, Wuxi, China. Diosmine and Diosmine mimetic agent are provided by NANJING CHIA TAI TIANQING Group Co. Ltd, Nanjing, China. The mimetic agent has the same shape, size, taste, colour, package and Lot number.

\section{Interventions}

\section{Liang-Xue-Di-Huang Decoction with the addition of Diosmine mimetic agent group}

Patients will take one Liang-Xue-Di-Huang Decoction per day, 2 times a day, be took 1 hour after lunch and dinner meals. Diosmine mimetic agent, $0.45 \mathrm{~g}$ each time, 2 times a day, be took 2 hours after lunch and dinner meals. The course of treatment will last 7 days, unless there is a loss of follow-up. Patients will be contacted by telephone in 7 and 14 days, and queried regarding adherence to study agents, illnesses, medication and supplement use. The assessment that needs to be performed at visit are listed in Figure 2.

\section{Diosmine with the addition of Liang-Xue-Di-Huang Decoction mimetic agent group}

Diosmine with the addition of Liang-Xue-Di-Huang Decoction mimetic agent group's treatments and measurements will be in accordance with Liang-Xue-Di-Huang Decoction with the addition of Diosmine mimetic agent group.

\section{Outcome measures}

The primary outcome measure of this study is the French Bleeding Score (Table 2) in 7 and 14 days. The Secondary outcome measure are Goligher Prolapse Score (Table 3) and Quality-of-Life Score (Table 4) in 
7 and 14 days[available online at www.jvir.org].

\section{Safety evaluation}

A blood routine examination, routine urine test, liver function test, renal function test, electrocardiograph, and urine pregnancy test (women only) will be administered for safety outcomes, which are monitored both before and after clinical intervention.

\section{Data management}

Information from the clinical examination, as well as evaluation of treatment efficacy, will be recorded in each patient's case report form (CRF). The study record is the source document of clinical study subject and should be kept in hospital. Each center will design designated personnel to be the electronic CRF input staff. Upon completion of each subject observation, the investigator will promptly submit the study record to the CRF inputter. The electronic CRF encoder must review whether the project record of the study notes is complete and report on time.

\section{Adverse events}

All adverse events, including toxicity and side effects, such as gastrointestinal reaction, liver damage, and renal failure will be recorded and graded in detail throughout the study. When a severe adverse event occurs, researchers will provide every necessary treatment, and report the adverse event to the ethic committee of Affiliated Hospital of Nanjing University of Chinese Medicine.

\section{Statistical analysis}

Frequency, median, and mean \pm standard deviation of the bleeding score, goligher prolapse score and quality-of-life score will be used for descriptive statistics. The statistical analysis will be performed using SAS 9.4. $P<0.05$ is considered statistically significant.

\section{Discussion}

HD is a well-defined clinical and pathophysiological placement between benign conditions but with high impact on quality of life $\mathrm{e}^{[12]}$. Chronic bleeding is the main symptom of $\mathrm{HD}^{[4]}$. It is estimated that $50 \%$ of people over 50 years of age have experienced symptoms of HD at least once in their life, and one-third of patients affected by HD seek medical attention ${ }^{[13,14]}$. Excessive bleeding can lead to an emergency situation. Hemorrhoidectomy and stapled hemorrhoidopexy are validated and effective surgical techniques, but are associated with long, painful postoperative courses with a significant complication rate, still required medical therapy ${ }^{[15]}$. Contrary to common belief, a nonsurgical treatment is quite effective to manage $\mathrm{HD}^{[16]}$, which can be offered with expectations of minimal harm and a decent potential for relief ${ }^{[6]}$, suggesting that medical therapy should be the first-line therapy for this disease ${ }^{[17]}$. 
Liang-Xue-Di-Huang Decoction has been used to treat HD from the 19th century in China. Over this period, rich experience in this medication has been accumulated. In 2018, National Administration of Traditional Chinese Medicine of People's Republic of China published 100 classic prescriptions of ancient Chinese medicine, which contained Liang-Xue-Di-Huang Decoction ${ }^{[9]}$. In this prescription, Sphora japonica $L$. (Huaijiao) and Platycladus orientalis(L.)Franco (Cebaiye) are the Jun (emperor) components in LiangXue-Di-Huang Decoction. Sphora japonica L. (Huaijiao), which was first recorded in Sheng Nong's herbal classic, is commonly applied in clinical practice for the treatment of hematochezia. It has the effect of cooling blood, stopping bleeding, clearing heat in bowels and eliminating swell and easing pain ${ }^{[18]}$. Modern pharmacological studies have demonstrated its efficacy for stopping bleeding and antiinflammation [19]. Platycladus orientalis(L.)Franco (Cebaiye) is categorized as a blood-cooling and hematostatic herb, which is usually prescribed with heat-clearing herbs to reinforce the efficacy of hemostasis. The pharmacological study of this herb validated its anti-inflammatory, antioxidant, antimicrobial activities, and considered as not toxic ${ }^{[20]}$. Sanguisorba officinalis L. (Diyu), Coptis chinensis Franch. (Huanglian), Rehmannia glutinosa (Gaetn.) Libosch. ex Fisch. et Mey. (Shengdihuang) are the the Chen (minister) components, synergize with Jun to strengthen its therapeutic effects. In traditional Chinese medicine (TCM), Sanguisorba officinalis L. (Diyu) is mixed with other herbs for the treatment of bleeding hemorrhoids. In vivo and in vitro studies have shown that Sanguisorba officinalis $L$. (Diyu) exhibited a wide range of pharmacological properties, including hemostatic, antibacterial activities $^{[21]}$. Coptis chinensis Franch. (Huanglian) has the effect of detumescence, clinically used for the treatment of hemorrhoid. Rehmannia glutinosa (Gaetn.) Libosch. ex Fisch. et Mey. (Shengdihuang) has been traditionally used as a blood cooling hemostatic. The Zuo (assistant) components, Angelica sinensis. (Danggui), CiTCMLIBus aurantium L. (Zhike), Scutellaria baicalensis Georgi. (Huangqin), Paeonia lactiflora Pall. (Chishao), Schizonepeta tenuifolia (Benth.) Briq. [Nepeta tenuifolia Bent.](Jingjie), and Trichosanthes Kirilowii Maxim. T. roswthornii Harms. (Tianhuafeng), activate blood circulation to remove stasis, eliminate possible adverse effects of the Jun and/or Chen components. The Shi (courier) components, Cimicifuga foetida L.[Actaea cimicifuga L.] (Shengma), and Radix Glycyrrhizae (Shenggancao) facilitate the overall action of the other components. Theoretically, Liang-Xue-Di-Huang Decoction work through the therapeutic principle Jun-Chen-Zuo-Shi, could not only directly relieve bleeding hemorrhoids but also diminish hemorrhoids prolapse.

Although TCM has been clinically practised for thousands of years, most Chinese herbal medicine products do not possess up-to-date data regarding their safety and modern scientific evidence for their claimed clinical uses. TCM safety research is becoming more standardized and is gradually aligning with international standards. A randomized, controlled, blind, and multicenter trial will be helpful for further prove the efficacy and safety of Liang-Xue-Di-Huang Decoction as an treatment for HD.

\section{Declarations}

\section{Ethical approval}


Central ethical approval has been confirmed from the group leader's ethic committee of Affiliated Hospital of Nanjing University of Chinese Medicine (ref approval no. 2019NL-158-02) and four sub-centers ethical will comply with the group leader's ethics approval. All patients have to obtain "Ethics approval and consent to participate" section and give their written informed consents prior to enrolment.

\section{Trial status}

The protocol version number is NO.2 and the date is January 10, 2019. At the time of manuscript submission, patient's recruitment for the trial is on-going. The clinical study will begin from Sept, 2019 and end in Dec, 2020. A total of 240 Chinese patients will be recruited in this clinical study.

\section{Funding}

This study is supported by the Key Laboratory of Famous Doctors' Proved Recipe Evaluation and Transformation Under State Administration of Traditional Chinese Medicine (NZYJDMF-2018002). This funding source provided test drugs, but it had no role in the design of this study and did not have any responsibility for analyses, interpretation of the data or the decision to submit results.

\section{Authors' contributions}

Qing Zhou and Shuo-yang Shi contributed to the design of the study protocol. Tuo Chen participated in the statistical design and helped in the design of the study. Ben-sheng Wu, Cheng-biao Xu, Ji Geng, Dan Zhang, Feng Jiang and Zong-qi He helped to draft the manuscript and participated in the project development. Yu-Gen Chen was the project leader for this research and participated in the critical revision of the manuscript. All authors have read and approved the final manuscript.

\section{Competing interests}

None.

\section{Abbreviation}

Hemorrhoidal disease $\quad H D$

Case report form $\quad$ CRF

Traditional Chinese medicine TCM

\section{Acknowledgements}

Not applicable.

\section{Consent for publication}

Individual participant image or other clinical detail is not applicable. 


\section{References}

[1] Jacobs DO.Hemorrhoids:what are the options in 2018?.Curr Opin Gastroenterol 2018,34:46-49.

[2] Altomare DF,Giuratrabocchetta S.Conservative and surgical treatment of haemorrhoids.Nat Rev Gastroenterol Hepatol 2013,10:513-521.

[3] Robert S,SandlerAnne F,Peery.Rethinking What We Know About Hemorrhoids CLIN GASTROENTEROL H 2019,17:8-15.

[4] G G Ravindranath,B G Rahul.Prevalence and risk factors of hemorrhoids:a study in a semi-urban centre.Int Surg J 2018,5:496-499.

[5] Wilson MZ,Swarup A,T Wilson LR,et al.The Effect of Nonoperative Management of Chronic Anal Fissure and Hemorrhoid Disease on Bowel Function Patient-Reported Outcomes.Dis Colon Rectum 2018,61:1223-1227.

[6] Davis BR,Lee-Kong SA,Migaly J,et al.The American Society of Colon and Rectal Surgeons Clinical Practice Guidelines for the Management of Hemorrhoids.Dis Colon Rectum 2018,61:284-292.

[7] Garg P.Conservative Treatment of Hemorrhoids Deserves More Attention in Guidelines and Clinical Practice.Dis Colon Rectum 2018,61:e348.

[8] Zagriadskiĭ EA,Bogomazov AM,Golovko EB.Conservative Treatment of Hemorrhoids:Results of an Observational Multicenter Study.Adv Ther 2018,35:1979-1992.

[9] National Administration of Traditional Chinese Medicine of People's Republic of China.Notice of National Administration of Traditional Chinese Medicine of People's Republic of China on the publication of the catalogue of 100 classic prescriptions of ancient Chinese medicine (the first batch) [EB/OL].http://kjs.satcm.gov.cn/zhengcewenjian/2018-04-16/7107.html

[10] Vijayananthan A,Nawawi O:The importance of Good Clinical Practice guidelines and its role in clinical trials.Biomed Imaging Interv J 2008,4:e5.

[11] State administration of traditional Chinese medicine.Criteria for diagnosis and efficacy of TCM diseases.Nanjing:nanjing university press 1994:132-133.

[12] Rubbini M,Ascanelli S,Fabbian F.Hemorrhoidal disease: is it time for a new classification? Int J Colorectal Dis 2018,33:831-833.

[13] Garg P.Novel Nonsurgical Treatment of Intractable Bleeding in Hemorrhoid Patients on Anticoagulants.Surg Innov 2018,25:423.

[14] Shelygin Y,Krivokapic Z,Frolov SA,et al. Clinical acceptability study of micronized purified flavonoid fraction 1000 mg tablets versus 500 mg tablets in patients suffering acute hemorrhoidal disease. CURR 
[15] Watson AJ,Hudson J,Wood J,et al.Comparison of stapled haemorrhoidopexy with traditional excisional surgery for haemorrhoidal disease (eTHoS):a pragmatic,multicentre,randomised controlled trial.Lancet 2016; 388:2375-2385.

[16] Garg P.Conservative Treatment of Hemorrhoids Deserves More Attention in Guidelines and Clinical Practice.Dis Colon Rectum 2018,61:e348.

[17] Wilson MZ,Swarup A,T Wilson LR,et al.The Effect of Nonoperative Management of Chronic Anal Fissure and Hemorrhoid Disease on Bowel Function Patient-Reported Outcomes.Dis Colon Rectum 2018,61:1223-1227.

[18] The State Pharmacopoeia Commission of PR China, Chinese Pharmacopoeia Commission, 2015 ed.,Beijing, 2015, http://www.drugfuture.com/Pharmacopoeia/CP2015-1/1642-1643.pdf.

[19] Shuang qin Wang,Jingjing Zhang,Juan Liu.et al.Quality evaluation of huaijiao pill by chromatographic fingerprint and simultaneous determination of its major bioactive components. Journal of Pharmaceutical Analysis 2016,4:249-255.

[20] Shan MQ,Shang, J,Ding AW.Platycladus orientalis Leaves:A Systemic Review on Botany,Phytochemistry and Pharmacology.AM J CHINESE MED 2014,42:523-542.

[21] Zhao Z,He X,Zhang Q,et al.Traditional Uses, Chemical Constituents and Biological Activities of Plants from the Genus Sanguisorba L.Am J Chin Med 2017,45:199-224.

\section{Tables}

Table 1 Standard formulation of Liang-Xue-Di-Huang Decoction

\begin{tabular}{|c|c|c|c|c|c|}
\hline Pinyin name & Latin name & Doses & $\begin{array}{l}\text { Pinyin } \\
\text { name }\end{array}$ & Latin name & Doses \\
\hline Huaijiao & Sphora japonica L. & $9 \mathrm{~g}$ & Cebaiye & $\begin{array}{l}\text { Platycladus } \\
\text { orientalis (L.)Franco }\end{array}$ & $6 g$ \\
\hline Diyu & Sanguisorba officinalis $L$. & $6 \mathrm{~g}$ & Huanglian & Coptis chinensis Franch. & $6 g$ \\
\hline Shengdihuang & $\begin{array}{l}\text { Rehmannia glutinosa } \\
\text { (Gaetn.) Libosch. ex Fisch. et } \\
\text { Mey. }\end{array}$ & $6 g$ & Danggui & Angelica sinensis. & $4.5 \mathrm{~g}$ \\
\hline Zhike & CiTCMLIBus aurantium L. & $3 g$ & Huangqin & $\begin{array}{l}\text { Scutellaria baicalensis } \\
\text { Georgi. }\end{array}$ & $3 g$ \\
\hline Chishao & Paeonia lactiflora Pall. & $3 g$ & Jingjie & $\begin{array}{l}\text { Schizonepeta tenuifolia } \\
\text { (Benth.) Briq. [Nepeta } \\
\text { tenuifolia Bent.] }\end{array}$ & $3 g$ \\
\hline Tianhuafeng & $\begin{array}{l}\text { Trichosanthes Kirilowii } \\
\text { Maxim.T. roswthornii Harms. }\end{array}$ & $2.4 \mathrm{~g}$ & Shengma & $\begin{array}{l}\text { Cimicifuga foetida L.[Actaea } \\
\text { cimicifuga L.] }\end{array}$ & $1.5 \mathrm{~g}$ \\
\hline Shenggancao & Radix Glycyrrhizae. & $1.5 \mathrm{~g}$ & & & \\
\hline
\end{tabular}


Table 2 French Bleeding Score (Possible Score=0-9)

Frequency

\begin{tabular}{cc} 
Never & 0 \\
\hline$>1$ per year & 1 \\
\hline$>1$ per month & 2 \\
$>1$ per week & 3 \\
\hline$>1$ per day or bowel movement & 4
\end{tabular}

\section{Bleeding}

Never

0

At wiping

1

In the toilet 2

On underwear 3

Anemia

Never

0

Without transfusion

1

With transfusion

2

\section{Table 3 Goligher Prolapse Score (Possible Score=0-3)}

Grade 1 No protrusion

0

Grade 2 Protrusion with spontaneous reduction 1

Grade 3 Protrusion requiring manual reduction 2

Grade 4 Protrusion that cannot be reduced

3

\begin{tabular}{lc}
\hline Table 4 Quality-of-Life Score & (Possible Score=0-4) \\
\hline No discomfort & 0 \\
Mild discomfort & 1 \\
Moderate discomfort & 2 \\
Significant discomfort & 3 \\
Permanent discomfort & 4 \\
\hline
\end{tabular}


Figures

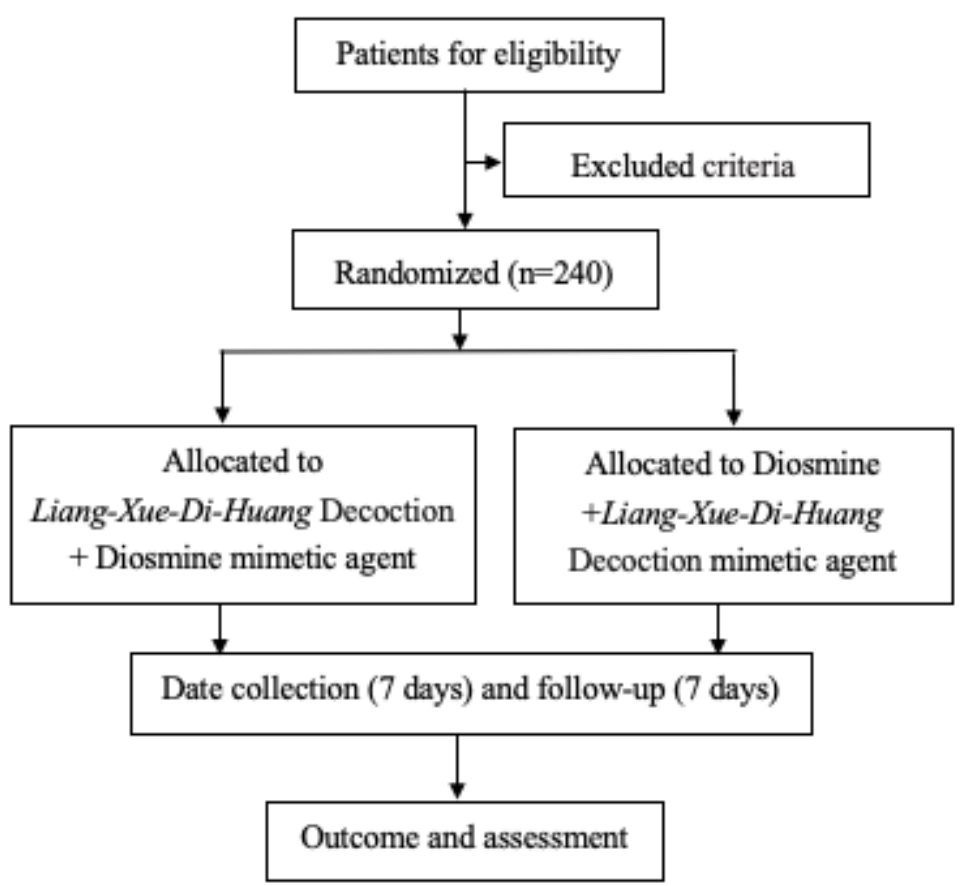

Figure 1

Study flow chart. The flow chart of enrolment, allocation, intervention and assessment. 


\begin{tabular}{|c|c|c|c|c|c|}
\hline \multirow[b]{3}{*}{ Visit } & \multicolumn{5}{|c|}{ Study period } \\
\hline & \multirow{2}{*}{$\frac{\text { Enrolment }}{\text { Visit } 1}$} & \multirow{2}{*}{$\begin{array}{c}\text { allocation } \\
\text { Visit } 2\end{array}$} & \multicolumn{2}{|c|}{ Post-allocation } & \multirow{2}{*}{$\frac{\text { Close-ou }}{\text { Visit } 4}$} \\
\hline & & & & Visit 3 & \\
\hline Time point & Day -1 & Day 0 & Day1 & Day 7 & Day 14 \\
\hline \multicolumn{6}{|l|}{ Enrollment } \\
\hline Informed consent form & $\bullet$ & & & & \\
\hline History & $\bullet$ & & & & \\
\hline Age & $\bullet$ & & & & \\
\hline Gender & $\bullet$ & & & & \\
\hline Inclusion criteria & $\bullet$ & & & & \\
\hline Exclusion criteria & $\bullet$ & & & & \\
\hline Randomisation and allocation & $\bullet$ & & & & \\
\hline Drug distribution & & $\bullet$ & & & \\
\hline \multicolumn{6}{|l|}{ Intervention } \\
\hline $\begin{array}{l}\text { Liang-Xue-Di-Huang Decoction } \\
\text { + Diosmine mimetic agent }\end{array}$ & & & • & $\bullet$ & \\
\hline $\begin{array}{l}\text { Diosmine } \\
\text { + Liang-Xue-Di-Huang } \\
\text { Decoction mimetic agent }\end{array}$ & & & $\bullet$ & $\bullet$ & \\
\hline \multicolumn{6}{|l|}{ Assessme } \\
\hline Ital signs & $\bullet$ & & & $\bullet$ & $\bullet$ \\
\hline Physical examination & $\bullet$ & & & $\bullet$ & $\bullet$ \\
\hline Blood routine examination & $\bullet$ & & & $\bullet$ & $\bullet$ \\
\hline Routine urine test & $\bullet$ & & & $\bullet$ & $\bullet$ \\
\hline Electrocardiograph & $\bullet$ & & & $\bullet$ & $\bullet$ \\
\hline Liver function test & $\bullet$ & & & $\bullet$ & $\bullet$ \\
\hline Renal function test & $\bullet$ & & & $\bullet$ & $\bullet$ \\
\hline Proctoscopy & $\bullet$ & & & $\bullet$ & $\bullet$ \\
\hline Urine pregnancy test (women) & $\bullet$ & & & $\bullet$ & $\bullet$ \\
\hline Bleeding score & $\bullet$ & & & $\bullet$ & $\bullet$ \\
\hline Goligher prolapse score & $\bullet$ & & & $\bullet$ & $\bullet$ \\
\hline Quality-of-life score & $\bullet$ & & & $\bullet$ & $\bullet$ \\
\hline
\end{tabular}

\section{Figure 2}

Study schedule for patients. After the enrolment and allocation, participants will receive a 7-day treatment and a 7-day follow-up. The time-points of assessment are shown in the schedule.

\section{Supplementary Files}


This is a list of supplementary files associated with this preprint. Click to download.

- supplement1.docx 